\title{
FAULT ANALYSIS IN BATTERY MODULE DESIGN FOR ELECTRIC AND HYBRID VEHICLES
}

\author{
B. Wu ${ }^{* 1}$, G. J. Offer $*^{1}$, V. Yufit ${ }^{1}$, D. A. Howey ${ }^{2}$ and N. P. Brandon ${ }^{1}$ \\ ${ }^{1}$ Department of Earth Science and Engineering, Imperial College London, London SW7 $2 A Z$ \\ ${ }^{2}$ Department of Engineering Science, University of Oxford, Oxford OX1 3PJ \\ *E-mail of corresponding author: billy.wu06@imperial.ac.uk
}

Keywords: Electric vehicle; Battery pack; Lithium battery; Fault finding; Battery management; Massively parallel pack

\begin{abstract}
In this paper systems integration issues, such as electrical and thermal design and management of full battery packs - often containing hundreds of cells - are discussed. The design and construction of a $9 \mathrm{kWh}$ battery pack for a motorsports application is used as an example. The pack contained 504 lithium cells arranged into 2 sidepods, each containing 3 modules, with each module in a $12 \mathrm{P} 7 \mathrm{~S}$ configuration. This paper focuses on describing problems related to cells being connected in parallel, known as massively parallel packs. We also demonstrate how a full vehicle test can be used to identify malfunctioning strings of cells for further investigation. It is shown that normal inter-cell contact resistances can cause currents to flow unevenly within the pack, leading to cells being unequally worked. This is supported by a Matlab/Simulink model of one battery module, including contact resistances, which was able to reproduce the results that were seen in experimental tests. Over time the unequal current flowing through cells can lead to significant differences in cells' state of charge and open circuit voltages, large currents flowing between cells even when the load is disconnected, and ultimately, some cells discharging and aging more quickly than others and jeopardising the energy storage capacity and lifetime of the entire pack.
\end{abstract}

\section{Introduction}

Electric and hybrid vehicles depend on electrochemical battery systems, with lithium-ion chemistries widely accepted as the current state of the art in terms of energy density and performance. Research and development into new electrode and electrolyte materials and chemistries continues apace. However, the electrical, mechanical and thermal integration of cells into packs and packs into electrical vehicles is paramount in order to ensure long and safe operation. Such integration issues have been relatively poorly researched in the literature to date. Whilst there are papers exploring the electrical and thermal behaviour of individual cells under a variety of conditions [1-5], the monitoring and testing of individual cells in battery packs $[6,7]$, and the thermal management of battery packs [4, 8-10], there are few which look at the electrical issues associated with the design and testing of complete modules, and those that do are for much lower power applications [11].

High power lithium-ion cells have very low internal resistances, of the order of $\mathrm{m} \Omega$, and the contact resistances between cells are normally measured in $\mu \Omega$. However, faulty contacts which lead to much higher contact resistances (e.g. of the order of $\mathrm{m} \Omega$ ) will adversely affect pack performance, often in unexpected ways, and this can be detected by current pulse techniques combined with individual cell testing, as described later in this paper. In parallel-connected cells, it is normally assumed that 'self balancing' will occur due to all cells being forced to the same potential [11]. However, as we later discuss, large differences in interconnection resistances in this configuration can lead to differences in the currents flowing through individual cells, which means that cells may not be well balanced under load and over time this could lead to unequal performance of individual cells. Clearly it is important to avoid this. In addition, if unequal current pathways are introduced through poor design cells may become unbalanced under load.

Previous authors have shown that short current pulses can be used to estimate the series resistance and other parameters of a battery system, and that the resulting current and voltage behaviour of the full pack in a real vehicle follows the model predictions reasonably accurately [12]. Indeed, in addition to traditional frequency response measurement techniques [7], pulse techniques have been used to estimate the internal resistance of lithium cells in a hybrid vehicle, and this can be used to provide a measure of battery degradation (capacity fade) over time [6].

In this paper we explore a methodology for detecting and analysing faults in complete battery modules at the pack and vehicle testing stage, by using a current pulse (or 'current interrupt') method for pack level, and electrochemical impedance spectroscopy for cell level tests. A more detailed analysis and discussion of the present work is presented in an extended journal paper by the same authors [13].

\section{Battery pack construction}

The battery pack used for experimental testing consisted of 504 4.8Ah (at a $0.5 \mathrm{C}$ discharge rate) Kokam Lithiumpolymer pouch cells with a maximum and minimum operating voltage limit of $4.2 \mathrm{~V}$ and $2.7 \mathrm{~V}$ respectively. The cells have a rated capacity of $17.8 \mathrm{Wh}$ each and continuous discharge current of 96 A with a peak current of $192 \mathrm{~A}$. 
These were divided into two sidepods containing 252 cells each, for use in a prototype electric racing vehicle. Each sidepod was further subdivided into three modules containing 84 cells each. The cells in each module were arranged in a $12 \mathrm{P} 7 \mathrm{~S}$ configuration, giving each module an operating voltage range of $18.9 \mathrm{~V}$ to $29.4 \mathrm{~V}$. Multiple cells connected in parallel and series which are treated as effectively one cell is known as a massively parallel pack. The three modules were connected in series giving a sidepod operating voltage range of $56.7 \mathrm{~V}$ to $88.2 \mathrm{~V}$.

A battery management system (BMS) produced by REAP systems Ltd., monitored 14 voltages and 7 temperatures of each parallel strip of cells with a sampling rate of $1 \mathrm{~Hz}$. Voltage measurements were made at the outer point on one side of each parallel strip and temperature measurements were made in the centre of a strip where the temperature is assumed to be highest.

The main mechanical components of the battery modules are shown in Figure 1. Two gridded polypropylene plates provided the cell support for each battery module at the centre of the cells. The mid-plates are the most structurally important part of the modules, supporting the bulk weight of the cells and provide the fundamental support structure that other elements are fixed to. Two end plates sat around the cell tabs. Aluminium bars provided the electrical connections between the individual cells.

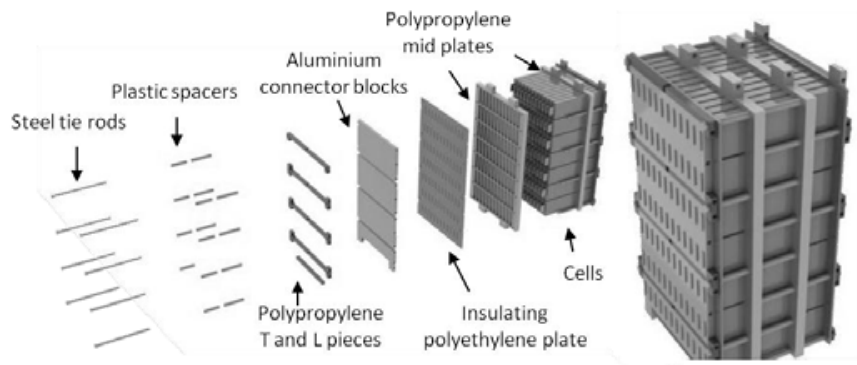

Figure 1: Exploded and fully assembled view of a battery module

The three modules were connected in series in each sidepod with electrical connections being made at the diagonal corners of the modules with bolted aluminium bars.

\section{Module testing and fault identification}

Testing was conducted on the battery modules in the vehicle configuration, i.e. the battery modules were installed on the vehicle and battery data was recorded during vehicle testing. The purpose of the testing was not to specifically test the battery packs, but to test the entire vehicle. The testing consisted of running the vehicle on a dynamometer and included acceleration events, i.e. brief bursts of high power, which was designed to stress the vehicle under simulated conditions representing extremes of operation.

\subsection{Acceleration test}

In the acceleration test the power was increased to $27.5 \mathrm{~kW}$ within 3 seconds, and then reduced to zero within 2 seconds.
An unusually high comparative voltage drop in one of the parallel strips in module 5 was noticed, which indicated a suspected fault. The current and voltage response measured using the BMS for module 5 is shown in Figure 2.

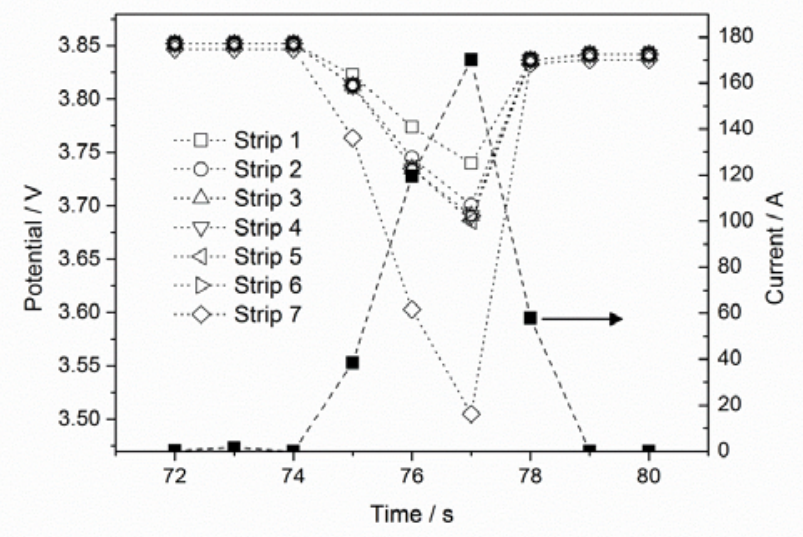

Figure 2: Test data from module 5 during an acceleration test on the dynamometer, current is shown with the axis on the right (black squares), and the voltage response of each strip of cells is shown with the axis on the left (hollow symbols)

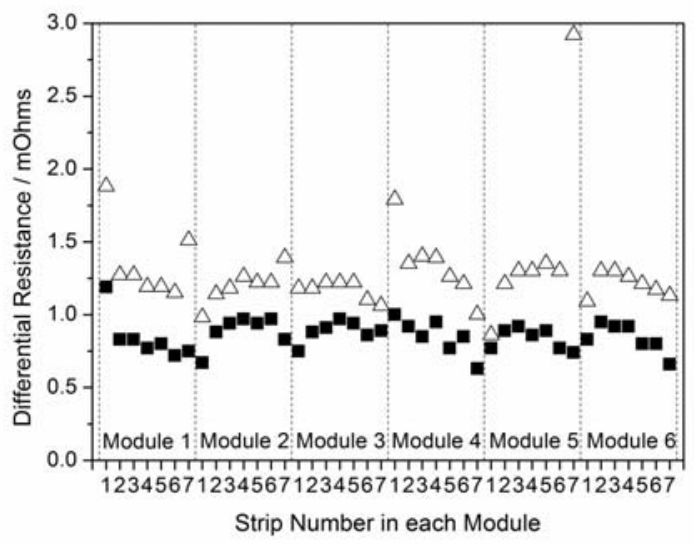

Figure 3: Differential resistance for all modules calculated from a current interrupt event with even contact resistances (filled squares), compared to the differential resistance for a 3 second current interrupt event with a single high contact resistance

The acceleration test can be likened to a current interrupt event, and therefore the data can be used to calculate a differential resistance $R$ using the equation $R=\Delta V / \Delta I$ between time stamp 77 and 78 where $\mathrm{R}$ is the differential resistance for a 1 second current interrupt, shown in Figure 3. This effectively lumps any processes with a frequency response of less than $1 \mathrm{~Hz}$ including all series resistances into a single resistance measurement. In practice this means that for a lithium-ion cell every dynamic process except diffusion and instantaneous ohmic voltage drop is not seen at this sampling rate [14].

\section{Identifying the fault cause}

It was hypothesised that the problem with module 5 strip 7 could either be caused by the cells, the module design or 
assembly. If the problems were caused by the cells then the cells in strip 7 of module 5 should have exhibited significant differences in their performance, which could have been caused either by manufacturing inconsistencies or damage to the cells at some point in their life. If the problems were caused by the module design or assembly then the unequal current paths must have been caused by a design flaw or high resistances introduced by substandard module components or contacts.

\subsection{Individual cell testing}

In order to test the first hypothesis that the problems were caused by the cells, the 12 cells from strip 7 of module 12 were individually tested by means of electrochemical impedance spectroscopy (EIS). The OCV at rest of the tested cells were $3.71 \mathrm{~V}$ which corresponded to $60 \%$ of SOC. The EIS spectra were recorded using an Autolab Potentiostat/FRA (Metrohm Inc) between 2000 and $0.1 \mathrm{~Hz}$ in galvanostatic mode with a current amplitude of $100 \mathrm{~mA}$. A typical example EIS spectrum of one of the individual cells and the equivalent circuit used to fit the data is shown in Figure 4.

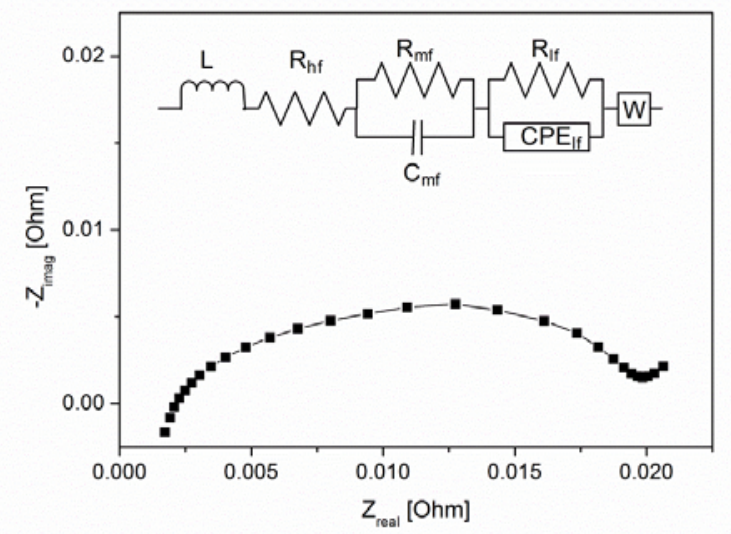

Figure 4: Typical EIS spectrum of the tested lithium polymer battery at OCV of $3.71 \mathrm{~V}$ and the equivalent circuit used to fit the data

Three processes can be distinguished and are fitted: 1) lithium ion transport through the Solid Electrolyte Interface (SEI) layer, 2) charge transfer at the electrode/electrolyte interface and 3) diffusion through a porous media. It is common to use an equivalent circuit comprising of an inductor, resistors, capacitors and so-called constant phase elements to fit the EIS response of an electrochemical device. The constant phase elements are used instead of capacitors when the EIS response in the complex plane represents a semi-depressed circle and have a general form of: $Z_{C P E}=(j * Q * \omega)^{-P}$ where $Q$ is a capacitance like parameter and $\mathrm{P}$ is a power index, usually between 0 and 1).

It was found experimentally that for this specific lithium polymer battery the best fit with lowest errors (up to 5\%) can be obtained with the equivalent circuit shown in Figure 4. The elements of this circuit are: high frequency inductor $\mathrm{L}$ and high frequency intercept $\mathrm{R}_{\mathrm{hf}}$ that arise as a result of the electrode and current collector electrical arrangement in the battery and series resistance of the current collectors, electrodes, electrolyte and connector terminals and wires respectively; mid frequency resistance $\mathrm{R}_{\mathrm{mf}}$ associated with the lithium ion transport through the SEI; low frequency resistance $\mathrm{R}_{\mathrm{lf}}$ related to the charge transfer; and generalised Warburg diffusion $\mathrm{W}$ through porous electrodes. The complex non-linear least squares parameter fit was carried out using ZView software (Scribner Inc). Note that $R_{m f}$ and $R_{l f}$ are also known as $R_{S E I}$ and $R_{c t}$ (charge transfer) respectively. All the cells had very similar fitted parameters and the values were well within the ranges expected from the manufacturer's data.

Results from the matching suggested the differences in various resistances among the cells was not significant enough to cause the measured fault. For example the difference between maximum and minimum values for $\mathrm{R}_{\mathrm{HF}}$, $\mathrm{R}_{\mathrm{CT}}, \mathrm{R}_{\mathrm{SEI}}$ and $\mathrm{R}_{\text {Total }}$ did not exceed $13.5 \%, 14 \%, 20 \%$ or $11 \%$ respectively, with mean values being $1.5,14,4$ and $18 \mathrm{~m} \Omega$ respectively. It should be noted that the values of series resistance did not indicate any problems as a result of possible corroded contacts, oxidised current collectors or poorly conductive solid polymer electrolyte in the tested cells. Neither did resistances of charge transfer and SEI transport. Hence, the battery module fault was not caused by any of the tested cells.

\subsection{Battery pack modelling}

In order to test the second hypothesis that the problems were caused by the module design or assembly, a model of a battery module was created in Simulink using the SimPowerSystems toolbox.

Using the Simulink battery model, the cells were arranged in a $12 \mathrm{P} 7 \mathrm{~S}$ configuration, with electrical connections being made at opposite corners of the module. A controlled current source connected between the positive and negative terminals of the module allowed for the regulation of module current from an inputted load cycle from a look-up table.

Each cell within the simulation was connected to a resistor at both its positive and negative terminals. This represents the contact resistance of the aluminium clamps to the cell tabs. Another resistance was then placed between each set of cells to represent the resistance of the aluminium interconnectors. This model therefore allows for the simulation of the effects of a higher contact resistance.

\subsubsection{Battery model}

The Simulink SimPowerSystems battery model was used to model the cells in the module. This model is based on a set of semi-empirical relationships and is able to characterise a number of different battery chemistries including: lead-acid, lithium-ion, nickel-cadmium and nickel-metal-hydride. By matching up the parameters in the model with the discharge characteristics of cells, representative behaviour of the pack could be simulated. 
Under low discharge rates $(<5 \mathrm{C})$ the battery model acceptably replicates the performance of the real cell with a reasonable degree of accuracy. At higher rates of discharge $(>10 \mathrm{C})$ the battery model becomes less accurate, but for the purpose of these simulations a discharge rate of $>10 \mathrm{C}$ was not be experienced and therefore this level of accuracy was deemed acceptable.

\subsubsection{Comparison of results}

Using the full battery module model, the current interrupt experiment, as shown in Figure 2, was recreated. Using the drawn current as the input and cell strip voltage measurements from the left side of the module, it was possible to recreate the same voltage characteristics observed by introducing a high contact resistance in the $7^{\text {th }}$ cell strip between the $2^{\text {nd }}$ and $3^{\text {rd }}$ cell of the parallel strip. The nominal interconnect resistance was estimated as being $0.1 \mathrm{~m} \Omega$ and the contact resistance $3 \mathrm{~m} \Omega$. In order to match the current interrupt test voltage measurements, the high interconnect resistance was estimated to be $2.5 \mathrm{~m} \Omega$. Simulation results were shown to be in good agreement with the experimental results. The model was then used to explore the effect of removing the high interconnector resistance, and the results of the current interrupt test were rerun to give the response of the cell with the normal resistance.

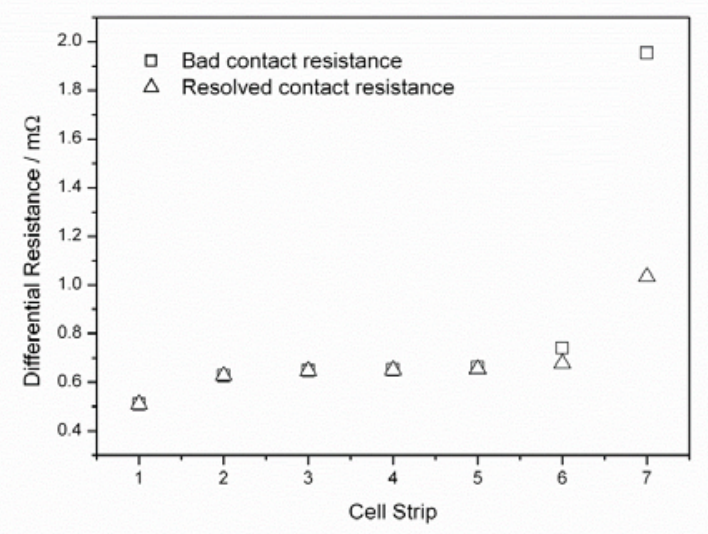

Figure 5: Differential resistance calculated from simulated current interrupt test of module 5 comparing the effects of a bad contact resistance and equal contact resistance

As shown in Figure 5, the presence of the high interconnector resistance leads to a high differential resistance being measured in cell strip 7, where the faulty connection was now suspected. By changing this back to the nominal resistance, the measured differential resistance returns to a normal value. In the case of equal contact resistances there is still an observed differential resistance due to the uneven current paths. This is a fundamental problem of massively parallel packs of this type.

The shape of the differential resistance curve, in Figure 5, across the cell strips is a sideways $\mathrm{S}$-shape. If the electrical connections were made on the same side of the module this would be a U-shaped curve. This effect can be explained using the model.

The current drawn from each individual cell was also simulated for even and uneven contact resistances. Figure 6 shows this current distribution for the module with oppositely connected electrical terminals with a single high contact resistance for a $150 \mathrm{~A}$ discharge just after the load has been applied.

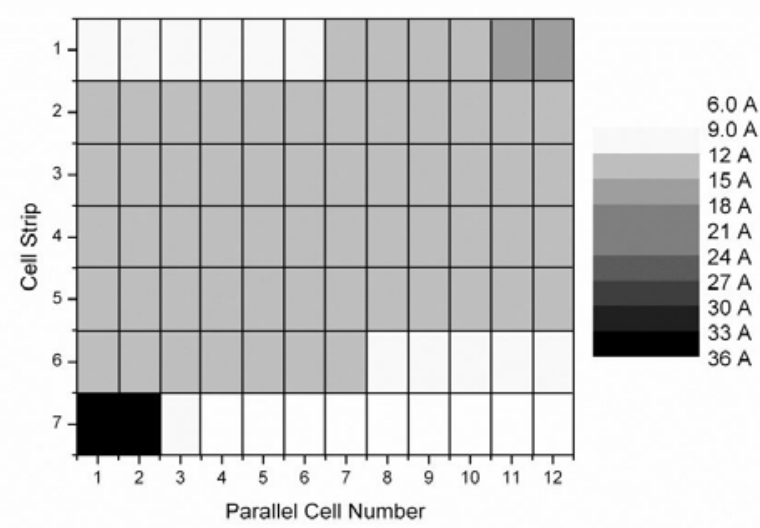

Figure 6: Simulated current distribution in battery module with a bad contact resistance for a 150A discharge

In Figure 6, the highest observed currents are at the electric connection points at opposite corners of the module, with the highest currents being observed diagonally across the module. The cells that are at the opposite corner, relative to the electrical connections, experience a much lower discharge current. This effect also occurs when the electrical connection points are on the same side. In the no fault case, the maximum discharge current is still double that of the minimum. This effect therefore leads to cell unbalancing and internal currents flowing when no load is applied after a load. When a high contact resistance is introduced, this restricts current flow from the cells isolated by the high contact resistance, drastically increasing the load on the cells near the current collection and decreasing the load on the cells isolated by the high contact resistance. Under the same discharge conditions, the model predicts the maximum discharge current to be 6 times that of the minimum discharge current. This highlights the importance of minimising contact resistances and ensuring equal balancing.

\section{Resolving the fault}

The most serious fault was identified to be in module 5 strip 7 , and it was suspected that either a single high contact resistance around cell 9 in the strip was effectively isolating 8 or 9 cells beyond the high contact resistance, or a combination of high resistances were causing a similar effect. This meant that the majority of the current was flowing through 3 or 4 cells nearest the current collection connection, causing the voltage drop across those cells to be significantly higher than in the other cells in that strip. As the BMS was monitoring the voltage across module 5 strip 7 on the same side at the current collection connection, it measured the 
higher voltage drop and not the lower voltage drop which it would measure if it was connected on the other side. This would give rise to a higher differential resistance measured by the current interrupt.

Therefore, module 5 was partially dismantled, to expose strip 7 , and the cell tabs and aluminium clamps were inspected for damage or anything that could cause a high resistance. A thin film of plastic, suspected to be glue, was found to be covering one side of the aluminium clamp between cells 8 and 9. The aluminium clamp was cleaned, and the module reassembled, put back in the vehicle, and then data from subsequent vehicle tests was analysed in order to check if the fault had been resolved. The module was then retested and the issue with the high differential resistance was resolved.

\section{Effect of using large capacity cells}

A possible solution to the problem of uneven current paths in massively parallel battery packs could be to use fewer high capacity cells to reduce the number of contact resistances, however this can actually exacerbate the problem. This is because with fewer but larger cells the resulting current has to be higher to achieve the same performance and therefore the effect of any faulty contact resistance is magnified. Figure 7 shows the current distribution for a simulated 6P7S pack configuration. The capacity of the cells was doubled to $9.6 \mathrm{Ah}$, such that the equivalent capacitance of the pack was the same, and the discharge curves matched up again.

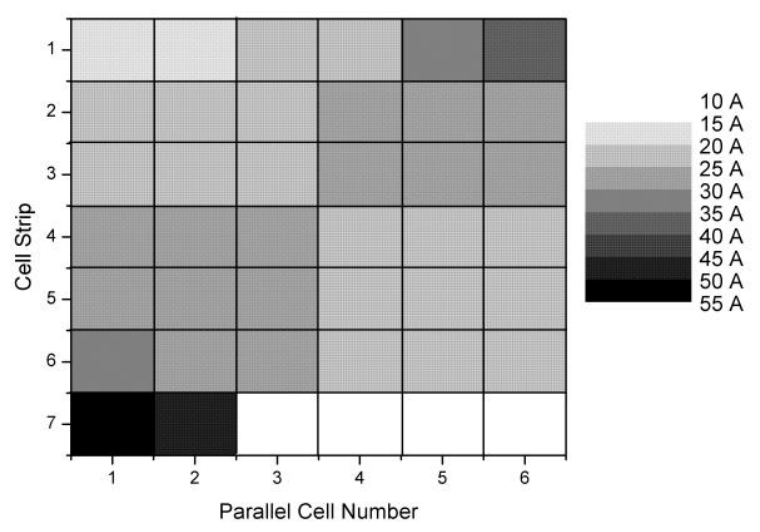

Figure 7: Simulated current distribution in 6PS7S battery module with $9.6 \mathrm{Ah}$ cells under a $150 \mathrm{~A}$ load with a high contact resistance at cell strip 7, cell number 2-3

As shown in Figure 7, the relative difference in current between the highest discharged cell and the lowest is roughly 5 times however the absolute difference is now as much as $45 \mathrm{~A}$ as opposed to $30 \mathrm{~A}$ in the $12 \mathrm{P} 7 \mathrm{~S}$ configuration.

Another problem with using larger cells in this case is that the relative discharge current, based on $\mathrm{C}$-rating, is less leading to smaller voltage variations. This would therefore make fault detection in a battery module using current interrupt techniques less effective. This is shown in Figure 8, where the simulation conditions were the same as shown in Figure 2.

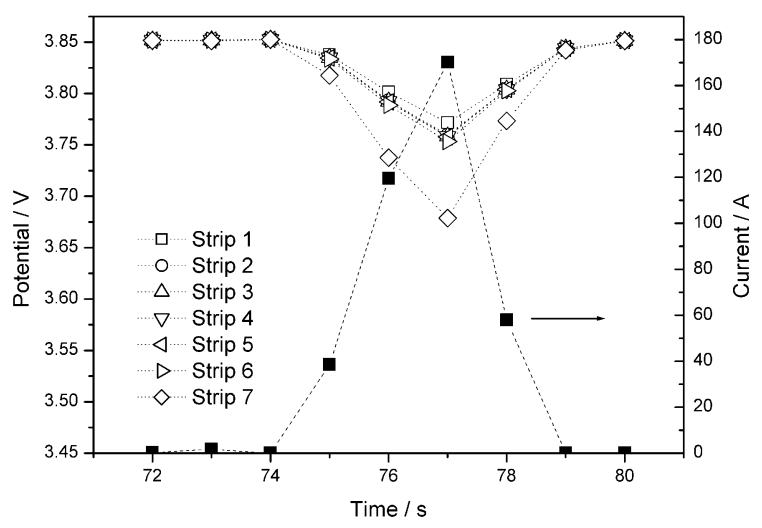

Figure 8: Simulated current interrupt test for a 7S6P battery pack with 9.6Ah cells, current is shown with the axis on the right (black squares), and the voltage response of each strip of cells is shown with the axis on the left (hollow symbols)

It therefore becomes apparent that the best solution would be to eliminate parallel strings. In the current work, the reason that a massively parallel design was chosen was because, under the competition rules for the particular vehicle, other configurations would have required the individual monitoring of all 504 cells in each module. For practical reasons this same reasoning might also dictate this design in many applications.

\section{Conclusion}

In this paper we have shown that, just as an individual cell may not behave uniformly under high current conditions, so an entire battery pack is also susceptible to non-uniform currents flowing through individual cells when there are parallel strings present in the pack, known as a massively parallel pack. This is particularly acute under high-C charge or discharge conditions. Even if the impedance of individual cells varies up to $10-20 \%$ this is not a cause of current flow non-uniformity across the battery modules. Instead, it is the interconnection resistances between highly paralleled cells which cause this effect.

We conclude that battery pack design must consider carefully designs which use highly paralleled strips of cells and also the electrical contact resistances present in the inter-connects between cells, and that anomalously high contact resistances can lead to serious imbalances in the way in which cells are used in the pack, with currents between parallel cells being unequal. A full pack test procedure can be used to identify cell strings which have much higher contact resistances.

We also conclude that for parallel connected cells there may be significant difference in cell states-of-charge after sustained continuous discharge if the cells are not carefully arranged such that equal currents flow through all of them. Even when equal currents do flow through cells, differences in temperature, voltage and other external and internal conditions across the cells may lead to differences in currents which become worse over time unless active monitoring or 
balancing is included. Upon returning to quiescent conditions, cells will tend to self-balance until parallel connecting strings have equal voltages. However, this may lead to large currents flowing and temperature increases.

The possibility of using larger capacity cells to have fewer contact resistances can in some cases exacerbate the problem of a single high contact resistance due to the increased current flow. The best solution would therefore be to have a single string of high capacitance cells to avoid the uneven current paths issue and therefore highlights to need for development of higher capacity cells.

There are some clear implications for the design of battery management and battery monitoring systems in this type of parallel arrangement. First, the BMS voltage sensing must located at side of module where the voltage drop is expected to be worst. Second, an active balancing BMS may need to include a delay before active balancing in order to allow time for passive balancing currents to flow within the pack.

\section{Acknowledgements}

The authors would like to thank all the students and supervisors involved in the Imperial Racing Green project who have been involved in the development of IRG04 the battery electric vehicle described in this paper. In particular the students who were involved in the design, making and testing of the IRG04 battery packs, Aran Kankiwala, Amit Meghani, Ian Hunt, Jessica Poore, Alice Rowlands, Aimen Sattar, Patrick White, Shahrukh Akhtar, Canupsorn Chairatna, Hassan Joudi, Lisheng Lu, Ashley Throop, Jielai Zhang, Ahmad Faiz Bakri, Chee Chung Lee, Shiang Jin Chin, Tian Yi Yuen, Yeit Hau Chin, David Topham, David Zhong, Thea Cooper and Rob Carter, and the supervisors who helped manage the students, Fred Marquis, Dave Robb, Graham Gosling, Paul Mitcheson, Finn Giuliani and Richard Silversides.

The authors would also like to thank the sponsors and supporters of Imperial Racing Green. In particular those who provided parts or sponsorship related to the development of the IRG04 battery packs, ABSL, Dow Kokam, and REAP Systems.

\section{References}

[1] J. Gomez, et al., "Equivalent circuit model parameters of a high-power Li-ion battery: Thermal and state of charge effects," Journal of Power Sources, vol. 196, pp. 4826-4831, May 152011.

[2] H. Vaidyanathan, et al., "Heat dissipation in a lithium ion cell," Journal of Power Sources, vol. 93, pp. 112-122, Feb 12001.

[3] C. Forgez, et al., "Thermal modeling of a cylindrical LiFePO(4)/graphite lithium-ion battery," Journal of Power Sources, vol. 195, pp. 2961-2968, May 1 2010.

[4] R. E. Gerver and J. P. Meyers, "Three-Dimensional Modeling of Electrochemical Performance and Heat
Generation of Lithium-Ion Batteries in Tabbed Planar Configurations," Journal of the Electrochemical Society, vol. 158, pp. A835-A843, 20112011.

[5] U. S. Kim, et al., "Effect of electrode configuration on the thermal behavior of a lithium-polymer battery," Journal of Power Sources, vol. 180, pp. 909-916, Jun 12008.

[6] J. Remmlinger, et al., "State-of-health monitoring of lithium-ion batteries in electric vehicles by on-board internal resistance estimation," Journal of Power Sources, vol. 196, pp. 5357-5363, Jun 152011.

[7] K. Takeno, et al., "Quick testing of batteries in lithium-ion battery packs with impedance-measuring technology," Journal of Power Sources, vol. 128, pp. 67-75, Mar 292004.

[8] K. Smith and C.-Y. Wang, "Power and thermal characterization of a lithium-ion battery pack for hybrid-electric vehicles," Journal of Power Sources, vol. 160, pp. 662-673, Sep 292006.

M. W. Verbrugge, "3-DIMENSIONAL TEMPERATURE AND CURRENT DISTRIBUTION IN A BATTERY MODULE," Aiche Journal, vol. 41, pp. 1550-1562, Jun 1995.

[10] T. M. Bandhauer, et al., "A Critical Review of Thermal Issues in Lithium-Ion Batteries," Journal of the Electrochemical Society, vol. 158, pp. R1-R25, 20112011.

[11] R. Spurrett, et al., "Modelling of highly-parallel lithium-ion batteries," in Proceedings of the Sixth European Space Power Conference. vol. 502, A. Wilson, Ed., ed, 2002, pp. 685-691.

[12] M. W. Verbrugge and R. S. Conell, "Electrochemical and thermal characterization of battery modules commensurate with electric vehicle integration," Journal of the Electrochemical Society, vol. 149, pp. A45-A53, Jan 2002.

[13] G. J. Offer, et al., "Module design and fault diagnosis in electric vehicle batteries," Journal of Power Sources, vol. accepted 12/01/2012, 2012.

[14] A. Jossen, "Fundamentals of battery dynamics," Journal of Power Sources, vol. 154, pp. 530-538, 2006. 